Check for updates

Cite this: RSC Adv., 2018, 8, 32211

Received 12th July 2018

Accepted 11th September 2018

DOI: 10.1039/c8ra05939j

rsc.li/rsc-advances

\section{Decontamination of radionuclides by functionalized mesoporous silica under gamma irradiation}

\author{
Sajid Iqbal ${ }^{\text {ab }}$ and Jong-II Yun (iD *a
}

Schiff base functionalized mesoporous silica (SA-SBA-15) was synthesized by the co-condensation method to remove the radioactive corrosion products from contaminated water coming from nuclear installations. SA-SBA-15 nanoparticles were first irradiated in a solid powder form and then applied to remove $\mathrm{Cu}(I), \mathrm{Ni}\left({ }^{\prime}\right)$, and Co(॥) from their aqueous mixture in the range from 0 to 1000 Gy of gamma irradiation. The FTIR, TGA, zeta potential, XRD, BET, TEM, and CHN analysis results revealed the stability of a ligand support material, mesopore ordering and the functional groups. The structural and functional group endurance under our studied gamma irradiation makes SA-SBA-15 a potential adsorbent for routine decontamination and decommissioning activities as well as in radioactively contaminated emergency scenarios. Furthermore, the adsorbent-metal complexation followed the Irving-Williams order $(\mathrm{Cu}(\|)>\mathrm{Ni}(\|)>\mathrm{Co}(॥))$ and showed a higher selectivity for $\mathrm{Cu}(॥)$ with removal capacity up to $99.76 \pm 0.01 \%$, thus suggesting its applicability to separate $\mathrm{Cu}(॥)$ from other metal ions.

\section{Introduction}

In nuclear power plants (NPPs), the removal of radioactive corrosion products is imperative to avoid intense occupational radiation exposure to plant workers, fuel heat transfer problems, and fuel failure. Activated corrosion products are released from several systems of NPPs such as steam generators, corroded pipes, and other structural materials into reactor coolant water. They can also be discharged into storage pond water from a corroded surface of spent fuel rods and waste streams coming from spilling and laundry decontamination.

The primary coolant system materials in pressurized water reactors (PWRs) are mainly composed of $\mathrm{Cr}-\mathrm{Ni}$-based steel alloys, Ni-based steel alloys, and Co-based steel alloys. Cu is also present in the system components as part of precipitation hardening stainless steels. The formation of process-related deposits of $\mathrm{Co}, \mathrm{Ni}$, and $\mathrm{Cu}$ can lead to an increase in primary water stress corrosion cracking on heat transfer surfaces and the resistance to heat transfer, resulting in fuel failure in NPPs. ${ }^{\mathbf{1 , 2}}$ Additionally, ${ }^{60} \mathrm{Co}$ is the major activated corrosion product responsible for gamma radiation build-up due to its high gamma energies (1.332 and $1.173 \mathrm{MeV}$ ) and relatively longer half-life (5.27 years).

Ion exchange resins (IEXs) are commonly used to purify the radioactively contaminated water and to maintain reactor

${ }^{a}$ Department of Nuclear and Quantum Engineering, KAIST, 291 Daehak-ro, Yuseong-gu, Daejeon 34141, Republic of Korea.E-mail: jiyun@kaist.ac.kr

${ }^{b}$ Chemistry Division, Pakistan Institute of Nuclear Science and Technology, P.O. Nilore, Islamabad, Pakistan coolant chemistry. During the entire decontamination process, IEXs remain under low and intermediate levels of gamma radiation and other environmental stresses, which may adversely affect their decontamination efficiency. Gamma irradiation forms different oxidative and reductive species by the radiolysis of water in the reactor coolant. The degradation of organic materials may occur through the excitation and ionization of absorbing atoms, and their subsequent chemical reactions can be influenced by free radicals and excited ions. Radiolysis and chemical degradation effects can be stronger and faster under the reactor coolant environment of high temperature and pressure. Gamma irradiations can affect the functional group and degrade the polymer linkage of resin leading to release of the captured radionuclides again into water. Generally, the absorbed gamma dose of $1 \times 10^{2}$ Gy does not affect the organic polymers significantly as mentioned in EPRI-NP-2129. ${ }^{3}$ Nevertheless, it is important to examine the gamma irradiation stability of the adsorbent if it is planned to apply for nuclear decontamination purposes. Up to now, numerous organic and inorganic resins have been investigated for decontamination of metal ions with different gamma irradiation stabilities from $10^{3}$ Gy to $10^{7} \mathrm{~Gy}$. For instance, poly-tetrafluoro-ethylene and aliphatic polyamide (nylon) bear the lowest threshold values of $1.5 \times 10^{2}$ and $8.7 \times 10^{2} \mathrm{~Gy}$, respectively, while glass-filled diallyl phthalate and asbestos phenolic laminate possess the highest stability against gamma absorbed dose of $1.8 \times 10^{9}$ and $1 \times 10^{9} \mathrm{~Gy}^{3}$

Mesoporous silica nanoparticles (MSNs) are promising materials due to their enormous applications in adsorption, biomedicine, and drug delivery. The hexagonal uniformly 
ordered mesoporous silica materials of MCM-41 (Mobil Composition of Matter no. 41) ${ }^{4}$ and SBA-15 (Santa Barbra Amorphous no. 15) ${ }^{5}$ were first produced in 1992 and 1998, respectively. They have received well deserved attention of researchers due to their high specific surface area $\left(>1000 \mathrm{~m}^{2}\right.$ $\left.\mathrm{g}^{-1}\right)$, tunable pore size, narrow pore diameter distribution, and excellent radiochemical and structural stability. MSNs are also known as popular ligand-solid supports for a selective removal of radionuclides during the separation/decontamination processes. MSNs can be synthesized by a variety of methods using large organic molecules unlike the conventional microporous zeolites and macroporous sponge-type materials. They are important for radioactive waste disposal applications due to the lower functional group steric hindrance, high chemical stability and non-swelling property. ${ }^{6}$ Different types of MSNs (SBA-15, MCM-41, and MCM-48 etc.) can be synthesized based on the variation of surfactants, the chemical ratio of precursors, synthesis temperature, and reaction time. SBA-15 is usually preferred over MCM-41 and MCM-48 as a solid support for ligand functionalization due to its large pore size $(5-30 \mathrm{~nm})$ and thick mesoporous walls (3-6 nm). The thicker walls provide an additional mechanical stability to SBA-15 compared to MCM-41 $(0.8-1.2 \mathrm{~nm})$ and MCM-48 $(0.8-1 \mathrm{~nm}) .{ }^{7,8}$ Another distinguishing feature of MSNs is the availability of surface active silanol groups $(\mathrm{OH})$, which can selectively be tailored to attach the desired functional groups/chelates at its surface. These chelates, such as amines, ethylenediaminetetraacetic acid (EDTA), diethylenetriaminepentaacetic acid (DTPA), imminodiacetic acid, thiols and carboxylic acids have already been incorporated to MSNs to enhance the adsorption capacity and selectivity for metal ions. ${ }^{9-13}$ Silica-based adsorbents have been tested to remove fission products such as Sr and Cs under high gamma irradiation. For example, Chen et al. used a silica-based adsorbent to selectively capture $\mathrm{Sr}$ (II) from a mixture of fission products as a function of temperature and gamma irradiations. ${ }^{14}$ Kikuchi and Suzuki studied the adsorption performance and structure stability of thiacalix[4] arene functionalized silica for americium removal under gamma irradiation..$^{15}$ These types of silica-based adsorbents can also be very useful to remove activated corrosion products from reactor coolant, spent fuel water tank and other waste streams generated from nuclear power installations.

Schiff's bases (SBs) are another class of versatile compounds having enormous applications in biology, chemotherapy, and adsorption. ${ }^{16}$ They can be readily synthesized by the condensation of primary amines with active carbonyl groups of aldehydes or ketones. ${ }^{17}$ SBs are synthesized using salicylaldehyde (SA) precursors that have significant complexation affinity for many transition metal ions such as $\mathrm{Cr}$ (III), $\mathrm{Mn}$ (II), $\mathrm{Fe}(\mathrm{III}), \mathrm{Ni}(\mathrm{II})$, and $\mathrm{Cu}(\mathrm{II}) .^{18}$ The SBs synthesized by aliphatic aldehyde precursors are relatively less stable and can easily undergo polymerization, whereas SBs synthesized using aromatic aldehydes (salicylaldehydes) are more stable due to the conjugation phenomenon. ${ }^{19,20}$ Additionally, the aromatic compounds are irradiation resistant and less sensitive to oxidation under harsh conditions. ${ }^{3}$ Therefore, it can be interesting to use SBs in adsorption study by fixing them at solid supports like mesoporous silica. In this way, SB complexed metals attached to solid silica support can be removed easily by centrifugation or filtration. Also it can be a promising inorganic-organic hybrid material for decontamination if the functionalized group remains stable in solids and in solutions under gamma irradiations.

The adsorbent should be resistant to gamma irradiations for the effective removal of radioactive corrosion products from nuclear reactor coolant and the wastes generated during power plant decontamination activities. Therefore, this study was typically designed to investigate the effect of gamma irradiations on the structural and functional group stability of SA-SBA15. To check the stability in a humid and oxidative environment, SA-SBA-15 was also irradiated in aqueous media in the presence of target metal ions.

\section{Materials and methods}

\subsection{Synthesis of pure SBA-15, $\mathrm{NH}_{2}-\mathrm{SBA}-15$, and SA-SBA-15}

SBA-15 and $\mathrm{NH}_{2}$-SBA-15 were synthesized by following a wellknown method reported elsewhere. ${ }^{21-23}$ Briefly, the SBA-15 surface silanol groups were coupled with an ethoxy group of aminopropyl tetraethoxysilane (APTES) to synthesize the aminefunctionalized mesoporous silica $\left(\mathrm{NH}_{2}\right.$-SBA-15), as illustrated in Fig. 1 (step 1). In the second step, the $N$-propylsalicyl-aldimino functionalized mesoporous silica (SA-SBA-15) was synthesized by the condensation of the surface primary amine group of $\mathrm{NH}_{2}$ SBA-15 with the carbonyl group of salicylaldehyde to form imine $(-\mathrm{C}=\mathrm{N}-)$, as shown in Fig. 1 (step 2). The condensation reactions were performed by refluxing a mixture of $\mathrm{NH}_{2}-\mathrm{SBA}-15$ and salicylaldehyde at $120{ }^{\circ} \mathrm{C}$ using an anhydrous toluene as a solvent under a perpetual inert atmosphere of argon. Yellowish precipitates were formed at the end of the condensation reaction after $12 \mathrm{~h}$. The obtained yellow slurry was recovered and washed many times with toluene, ethanol and dried overnight at room temperature. It was then dried at $60{ }^{\circ} \mathrm{C}$ under a vacuum for $8 \mathrm{~h}$ and stored in a sealed glass bottle for gamma irradiation testing, characterization and adsorption experiments.

\subsection{Characterization}

Attenuated total reflectance-Fourier transform infrared spectroscopy (ATR-FTIR) was used to obtain structural information about the functionality of synthesized adsorbents. The weight percentages of the $\mathrm{C}, \mathrm{H}$, and $\mathrm{N}$ atoms were determined by an elemental analyzer EA2 (Thermo Finnigan, Italia). A small-angle X-ray diffractometer (RIGAKU, D/MAX-2500, Japan) was utilized to record the diffraction patterns by using $\mathrm{Cu} \mathrm{K}_{\alpha}$ radiation $(\lambda=$ $1.5408 \AA$ ) with a scan rate of 0.6 to $5^{\circ}(2 \theta)$ and a resolution of $0.02^{\circ}$. The surface charge of both the bare and functionalized SBA-15 was measured by the zeta potential and particle size analyzer (ELS-Z2, Otsukael, Japan). A thermogram showing the weight loss with temperature was obtained by a thermogravimetric analyzer (TGA), (TGA 92-18, Setaram France). Highresolution transmission electron microscopy (HR-TEM) images were recorded on the Cs-corrected STEM (JEM- 


\section{STEP\#1}

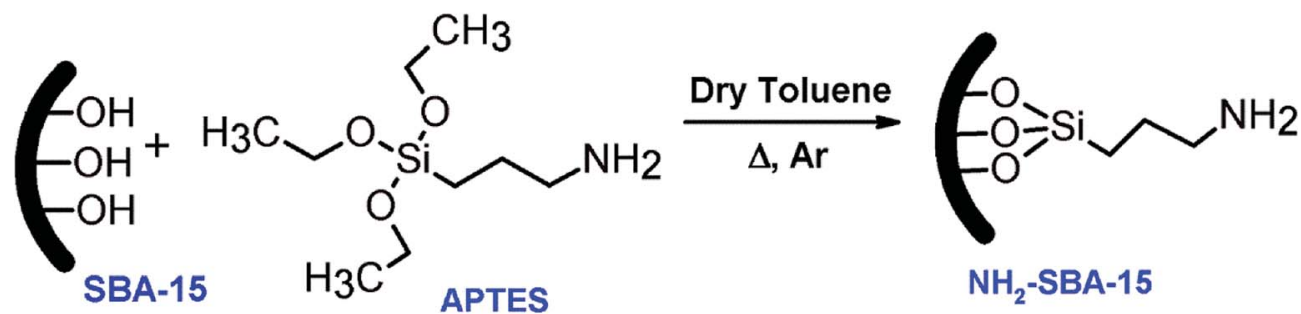

STEP\#2

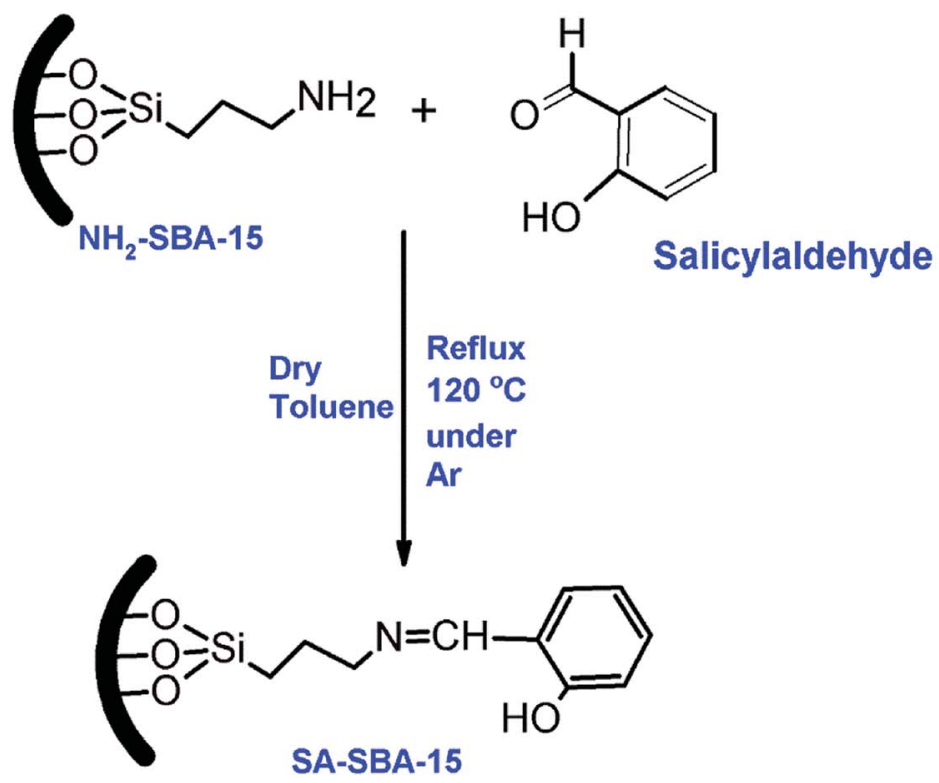

Fig. 1 Schematic of the stepwise functionalization scheme of SBA-15. Step \# 1 shows the amine grafting onto SBA-15; step \# 2 shows the coupling of the terminal amine with the salicylaldehyde by the co-condensation method.

ARM200F, JEOL, USA). The specific surface area and mesoporosity were measured by using the Brunauer-Emmett-Teller (BET) technique. The single point total mesopore volume was measured from the amount of nitrogen absorbed at relative pressure $\left(P / P_{\mathrm{o}}=0.99\right)$. Pore size distribution curves were acquired by the Barrett-Joyner-Halenda (BJH) method. A diodearray UV-Vis absorption spectrometer (ZEISS, MCS601 UV-NIR C, Germany) was employed to quantify the cobalt, copper, and nickel metal ions. Gamma irradiation experiments were performed by using Co-60 gamma irradiator at the Korea Atomic Energy Research Institute (KAERI).

\subsection{Adsorption and quantitative analysis}

Gamma irradiation experiments on a mixture of metal ions and adsorbent in a solution were performed after optimization of $\mathrm{pH}$ and solid to liquid ( $\mathrm{S}: \mathrm{L}$ ) ratio to ensure the maximum uptake of metal ions. Sorption capacity $\left(\mathrm{mmol} \mathrm{g}^{-1}\right)$ was analyzed as a function of $\mathrm{pH}$ from 1 to 6 at a fixed $\mathrm{S}: \mathrm{L}$ ratio of $1 \mathrm{~g} \mathrm{~L}^{-1}$ and $1 \mathrm{mM} \mathrm{Co(II)}$ at $150 \mathrm{rpm}$ shaking for $2 \mathrm{~h}$. The $\mathrm{S}: \mathrm{L}$ ratio was optimized at $\mathrm{pH} 6$. The ionic strength was maintained at $0.1 \mathrm{M}$ by $\mathrm{HCl} / \mathrm{NaCl}$. After achieving equilibrium in $2 \mathrm{~h}$, the supernatant was separated from adsorbent with $20 \mathrm{~nm}$ inorganic membrane filter (Whatman product no: 6809-1002) followed by 15 min centrifuge at $4000 \mathrm{rpm}$.

The quantitative analysis was performed by UV-Vis absorption spectrometer after overnight chelation of metal ions with $2.5 \times 10^{-5} \mathrm{M}$ Br-PADAP (2-(5-bromo-2-pyridylazo)-5diethylaminophenol) as a colorimetric reagent. The Br-PADAP solution was prepared with an equal volume ratio $(1: 1)$ of methanol and de-ionized water. The standard solutions of 0.1 to $1.0 \mathrm{mmol} \mathrm{L}^{-1}$ for each metal ion were prepared for method validation. The molar absorptivity was measured corresponding to the UV absorption bands of $\mathrm{Cu}$ (II) at $558 \mathrm{~nm}, \mathrm{Ni}(\mathrm{II})$ at $557 \mathrm{~nm}$, and $\mathrm{Co}$ (II) at $589 \mathrm{~nm}$ by following Beer-Lambert's law i.e. $A=\varepsilon c l$. $A$ is the absorbance, $\varepsilon$ is the molar absorptivity $\left(\mathrm{M}^{-1} \mathrm{~cm}^{-1}\right), c$ is the concentration (M) of target metal ion, and $l$ is the absorption path length of $1 \mathrm{~cm}$ in this study. The moral absorptivity $\varepsilon$ was obtained from the slope of calibration between 
concentration and absorbance drawn for each metal ion using corresponding standard solutions. Percentage removal $(\% R)$ of metal ions from their mixture was calculated by eqn (1) and the equilibrium sorption capacity $\left(q_{\mathrm{e}}\right)$ was measured by the formula shown in eqn (2)

$$
\begin{gathered}
\% R=\left(C_{\mathrm{o}}-C_{\mathrm{e}}\right) / C_{\mathrm{o}} \times 100 \\
q_{\mathrm{e}}=\left(C_{\mathrm{o}}-C_{\mathrm{e}}\right) \times V / m
\end{gathered}
$$

where $C_{\mathrm{o}}$ and $C_{\mathrm{e}}$ are the initial and the final metal ion concentration in $\mathrm{mmol} \mathrm{L}^{-1} . V$ and $m$ are the volume $(L)$ of solution and the mass of sorbent $(\mathrm{g})$ used for sorption process, respectively.

\subsection{Gamma irradiations set up}

In a practical scenario, SA-SBA-15 was applied to remove metal ions from a mixture under the gamma irradiation environment. Therefore, it was necessary to investigate the structural and functional group stability, and the adsorption behavior under gamma irradiations. After irradiation of solid nano-powder of SA-SBA-15, four batches containing $\mathrm{Cu}(\mathrm{II}), \mathrm{Ni}$ (II) and $\mathrm{Co}$ (II) samples ( $1 \mathrm{mM}$ of each metal ions) were prepared and stored in the pyrex bottles. The bottle's lid was closed tightly and wrapped with Teflon tape before delivering them to the gamma irradiation facility. Each sample set was exposed to absorb a total gamma dose of 1, 10, 100 and $1000 \mathrm{~Gy}$. The gamma dose rate and the total absorbed dose was adjusted by changing the sample distance from the Co-60 source, as shown in Fig. 2. The details of Co-60 gamma-ray source and irradiation conditions are reported in Table 1.

\section{Results and discussions}

The synthesized SA-SBA-15 (nano powder) was exposed to the range of gamma-ray doses $(0,1,10,100,1000 \mathrm{~Gy})$ to investigate its structural and functional group stability. The integrity of the functional group before and after gamma irradiation was assessed by the ATR-FTIR technique, and the results can be seen in Fig. 3. The stretching vibration at $1650 \mathrm{~cm}^{-1}$ (main band for Schiff's base chelate) was allocated to the $-\mathrm{C}=\mathrm{N}$ of the imine group. $^{24}$ The IR bands in the range of $1275-1281 \mathrm{~cm}^{-1}$ and $1618-1631 \mathrm{~cm}^{-1}$ were assigned to phenolic $(-\mathrm{C}-\mathrm{OH})$ and $(\mathrm{C}-\mathrm{N})$, respectively. ${ }^{2,25}$ From the FTIR spectra, it was found that the transmittance peaks of all the functional groups are located in the same position without any peak shift by increasing gamma doses ( 0 to $1000 \mathrm{~Gy}$ ). This irradiation stability of the functional group may be attributed to a conjugation due to the aromaticity of aldehyde precursor (salicylaldehyde). ${ }^{3}$

To confirm the presence of the functional group and to propose the mechanism of functionalization, the molar concentrations of carbon and nitrogen were acquired from the $\mathrm{C} \mathrm{H} \mathrm{N}$ elemental analysis technique and the results are presented in Table 2 . It could be observed that the $\mathrm{C} / \mathrm{N}$ ratios in $\mathrm{SA}$ SBA-15 appeared in the range of $9.60 \pm 1.81$ to $10.13 \pm 0.53$ under absorbed gamma irradiations ( 0 to $1000 \mathrm{~Gy}$ ), whereas the theoretically calculated $\mathrm{C} / \mathrm{N}$ ratio was 10.0 . This shows close agreement of our experimental results with the theoretical calculation. Based on the information, the 1:1 complexing stoichiometry between $\mathrm{NH}_{2}$-SBA-15 and the salicylaldehyde can be suggested, which is in good agreement with the results reported by Mureseanu et al. ${ }^{24}$ Moreover, the loading of SA (1 molecule per $\mathrm{nm}^{2}$ ) onto the SBA-15 surface was calculated by taking into account the specific surface area. This can be explained by the known available silanol $(-\mathrm{OH})$ concentration at SBA-15. The silanol groups (3.31 molecules per $\mathrm{nm}^{2}$ ) at SBA-15 have been reported by using nuclear magnetic resonance (NMR) spectroscopy and thermogravimetric techniques. ${ }^{26}$ From Fig. 1, it can be seen that three silanol groups are linked with three ethoxy groups $\left(-\mathrm{OC}_{2} \mathrm{H}_{5}\right)$ of APTES during $\mathrm{NH}_{2}$-SBA-15 synthesis (Fig. 1). The experimental and theoretical $\mathrm{C} / \mathrm{N}$ ratio suggests the 1:1 stoichiometry between the amino and SA group in SA-SBA-15.

The SA grafting onto the SBA-15 surface was also confirmed by thermogravimetric analysis (TGA), as shown in Fig. 4. The effect of physisorbed water $(2.8 \%$ in SBA-15 and $2.1 \%$ in SA-SBA-

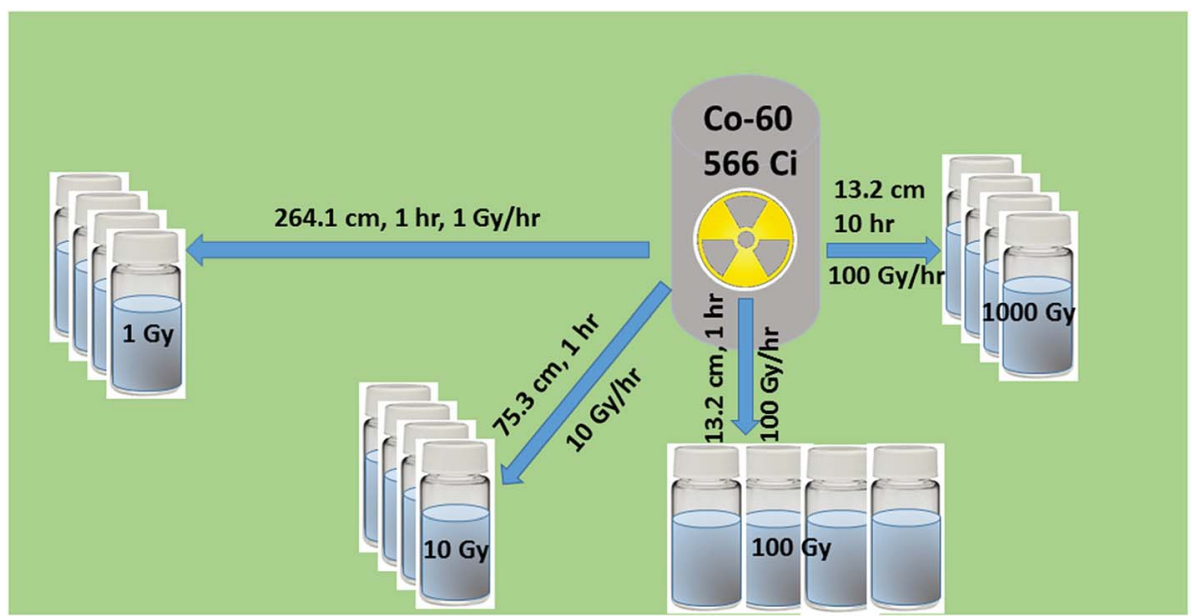

Fig. 2 Schematic of the gamma irradiation process. Gamma irradiation dose was adjusted by varying the distance from the Co-60 source and the dose rate. 
Table 1 Gamma irradiation conditions for SA-SBA-15 in powder and liquid mixed forms

\begin{tabular}{ll}
\hline Irradiation type & Gamma ray \\
Irradiation source & Co-60 \\
Source activity & $566 \mathrm{Ci}$ \\
Energy spectrum & $1.17,1.33 \mathrm{MeV}($ avg. $1.25 \mathrm{MeV})$ \\
Total absorbed dose & 1.0 to $1.0 \times 10^{3} \mathrm{~Gy}$ \\
Absorbed dose rate & 1.0 to $1.0 \times 10^{2} \mathrm{~Gy} \mathrm{~h}^{-1}$ \\
Irradiation time & 1 to $10 \mathrm{~h}$ \\
Irradiation temperature & $25^{\circ} \mathrm{C}$
\end{tabular}

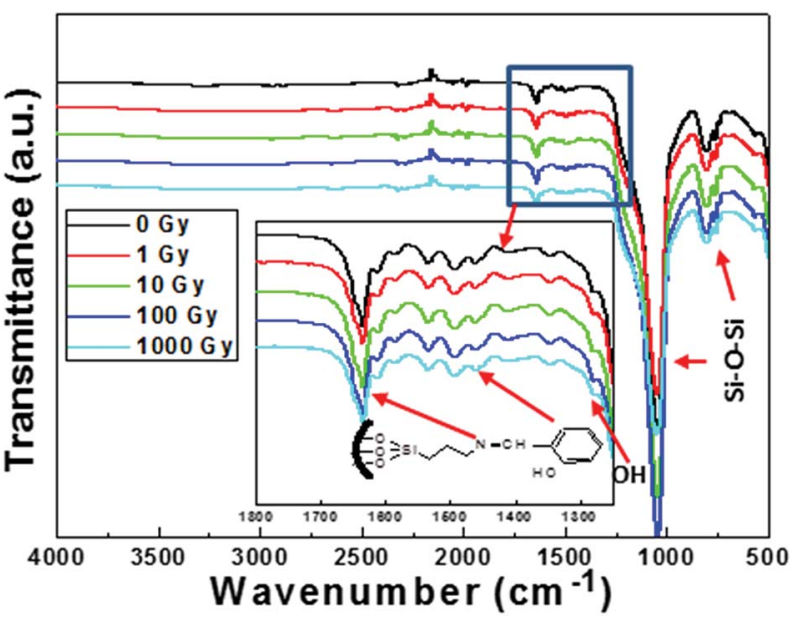

Fig. 3 FTIR spectra of the unirradiated and gamma irradiated SA-SBA15.

15) was examined up to $200{ }^{\circ} \mathrm{C}$. By comparing the SBA-15 and SA-SBA-15 thermograms, no significant weight loss was observed in SBA-15 from 200 to $700{ }^{\circ} \mathrm{C}$, whereas $10.2 \%$ weight loss was measured in the case of SA-SBA-15. The weight loss from $200{ }^{\circ} \mathrm{C}$ to $700{ }^{\circ} \mathrm{C}$ is due to the degradation of the immobilized functional group at the SBA-15 surface. ${ }^{27}$ Therefore, the SA grafting onto the SBA-15 surface was successfully confirmed.

Further confirmation of the SBA-15 surface modification was assessed by measuring the zeta potential of both the functionalized and bare SBA-15. From the results in Fig. 5, a significant difference among the zeta potential values was observed. Since the silica surface has a point of zero charge (pzc) at $\mathrm{pH} 2,{ }^{28}$ its surface comprises the negative charge $\left(\mathrm{Si}-\mathrm{O}^{-}\right)$at $\mathrm{pH}>\mathrm{pH}_{\mathrm{pzc}}$ and the positive charge $\left(\mathrm{Si}-\mathrm{OH}_{2}{ }^{+}\right)$at $\mathrm{pH}<\mathrm{pH}_{\mathrm{pzc}}$. In SA-SBA-15, the phenolic group $(\mathrm{OH})$ and an electron lone pair at $\mathrm{N}$ in the imine group get protonated due to the excess protons at a lower $\mathrm{pH}$

Table 2 Carbon and nitrogen concentrations as measured by the $\mathrm{C}$ $\mathrm{H} \mathrm{N}$ elemental analysis technique

\begin{tabular}{lllr}
\hline Gamma dose (Gy) & $\mathrm{N}\left(\mathrm{mmol} \mathrm{g}^{-1}\right)$ & $\mathrm{C}\left(\mathrm{mmol} \mathrm{g}^{-1}\right)$ & \multicolumn{1}{c}{$\mathrm{C} / \mathrm{N}$} \\
\hline 0 & $1.24 \pm 0.00$ & $11.92 \pm 0.01$ & $9.60 \pm 1.81$ \\
1 & $1.21 \pm 0.06$ & $11.63 \pm 0.09$ & $9.58 \pm 1.60$ \\
10 & $1.15 \pm 0.05$ & $11.66 \pm 0.03$ & $10.15 \pm 0.53$ \\
100 & $1.14 \pm 0.09$ & $11.92 \pm 0.04$ & $10.44 \pm 0.44$ \\
1000 & $1.17 \pm 0.05$ & $11.86 \pm 0.02$ & $10.13 \pm 0.53$
\end{tabular}

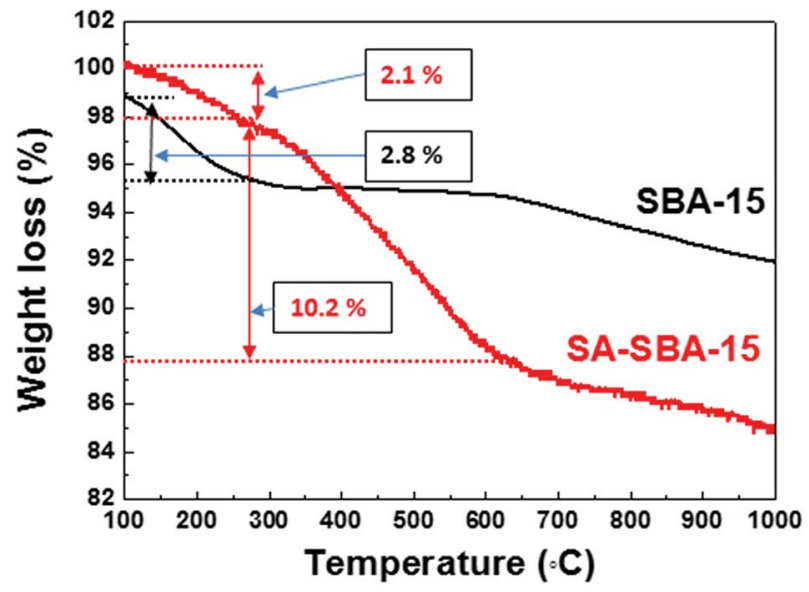

Fig. 4 TGA thermograms of SBA-15 and SA-SBA-15.

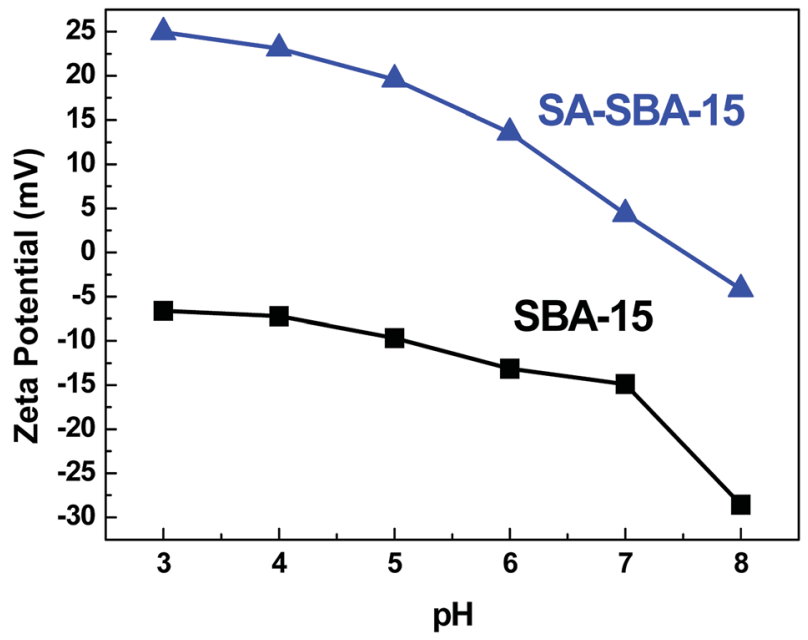

Fig. 5 Zeta potentials of SBA-15 and SA- SBA-15 as a function of $\mathrm{pH}$.

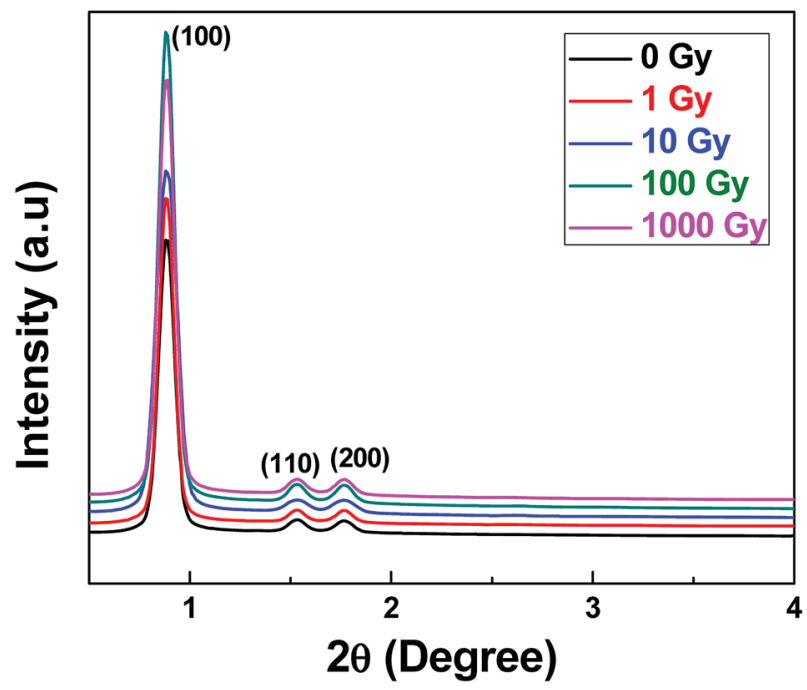

Fig. 6 X-ray diffraction patterns of unirradiated and gamma irradiated SA-SBA-15. 


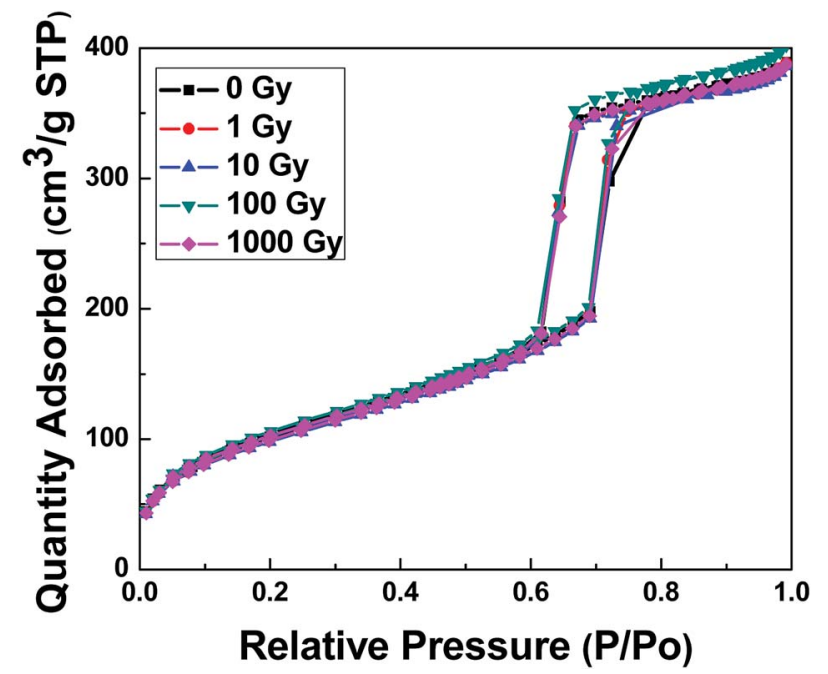

Fig. $7 \mathrm{~N}_{2}$ adsorption/desorption isotherms of non-irradiated and gamma-irradiated SA-SBA-15.

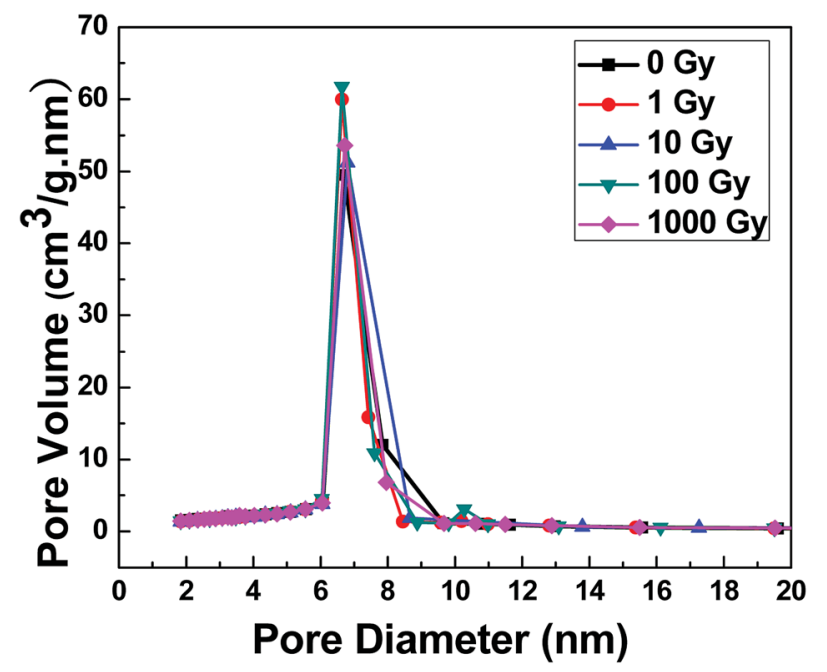

Fig. 8 Pore size distribution of non-irradiated and gamma-irradiated SA-SBA-15 calculated using the BET technique.

and become inaccessible for complexation, as reported in the literature. ${ }^{24}$ This protonation phenomenon could also be the probable reason for high zeta potential at a lower $\mathrm{pH}$ in SA-SBA15. Hence, the zeta potential results of pure and functionalized
SBA-15 also confirmed the successful loading of functional groups onto SBA-15.

The structural composition of SA-SBA-15 before and after gamma irradiations was confirmed by XRD, as shown in Fig. 6. Three distinct peaks at $2 \theta=0.89,1.54$ and 1.77 can be observed in all the XRD patterns. It is worth-noting that no peak shift was observed in the XRD patterns with gamma irradiations. Hence, there is no swelling/contraction that occurred with the absorbed gamma dose. This further confirms the FTIR results suggesting the functional group's stability under gamma irradiations.

The nitrogen adsorption/desorption isotherms and average pore size distributions are given in Fig. 7 and 8, respectively. According to the IUPAC classification of porous materials, an appearance of the hysteresis loop in the relative pressure range from 0.45 to 0.8 is an indication of mesoporous materials. ${ }^{29,30}$ (see Fig. 7) No effect of gamma irradiations was observed on the physisorption isotherms in all samples. The results also showed that the material preserved its mesoporosity even under gamma irradiations. Similarly, no significant change was observed in the pore size, as illustrated in Fig. 8, which proves the intactness of pores. The structural parameters such as the specific surface area $\left(S_{\mathrm{BET}}\right)$, the average pore size $\left(D_{\mathrm{p}}\right)$, the pore volume $\left(V_{\mathrm{p}}\right)$, and the pore wall thickness $\left(t_{\mathrm{w}}\right)$ were calculated and are shown in Table 3. Hence, all the structural parameters' values remained very close to each other with the increase in gamma irradiations.

The structural and pore morphology of non-irradiated and irradiated SA-SBA-15 were investigated by HR-TEM, and the results are given in Fig. 9. The top view surface TEM images show a honeycomb-like mesoporous structural morphology. No pore widening was observed under gamma irradiations. The mesopores are very uniform with an average diameter of $\sim 6.5 \mathrm{~nm}$ (in the magnified image in Fig. 9). The side view morphology of SA-SBA-15 shows a highly parallel and uniform channel-like morphology in Fig. 10. Similarly, no channel distortion/widening was observed under gamma irradiations. The average wall thickness of SA-SBA-15 is $\sim 3 \mathrm{~nm}$, while the average width of the channel is around $\sim 6.7 \mathrm{~nm}$. As shown in Fig. 9, the diameter of pores was further confirmed.

\subsection{Adsorption and irradiation of studies of SA-SBA-15}

The effect of $\mathrm{pH}$ and the solid to liquid $(\mathrm{S}: \mathrm{L})$ ratio were studied to optimize the maximum uptake of metal ions onto SA-SBA-15. $\mathrm{pH}$ is an important parameter to study the effect of surface

Table 3 Comparison of the structural parameters of non-irradiated and gamma-irradiated SA-SBA-15 ${ }^{a}$

\begin{tabular}{llllll}
$\begin{array}{l}\text { Irradiation } \\
\text { dose }(\mathrm{Gy})\end{array}$ & $S_{\mathrm{BET}}\left(\mathrm{m}^{2} \mathrm{~g}^{-1}\right)$ & $D_{\mathrm{p}}(\mathrm{nm})$ & $V_{\mathrm{p}}\left(\mathrm{cm}^{3} \mathrm{~g}^{-1}\right)$ & $d_{100}(\mathrm{~nm})$ & $a_{\mathrm{o}}(\mathrm{nm})$ \\
\hline 0 & $340 \pm 39$ & $6.25 \pm 0.05$ & $0.57 \pm 0.01$ & 10.0 & 11.6 \\
1 & $333 \pm 35$ & $6.35 \pm 0.15$ & $0.57 \pm 0.02$ & 10.0 & 5.3 \\
10 & $329 \pm 37$ & $6.40 \pm 0.10$ & $0.57 \pm 0.02$ & 10.0 & 11.6 \\
100 & $345 \pm 35$ & $6.40 \pm 0.10$ & $0.57 \pm 0.03$ & 10.0 & 11.6 \\
1000 & $352 \pm 18$ & $6.40 \pm 0.10$ & $0.54 \pm 0.04$ & 9.9 & 5.1 \\
\end{tabular}

${ }^{a} S_{\mathrm{BET}}=$ BET specific surface area, $D_{\mathrm{p}}=$ pore diameter, $V_{\mathrm{p}}=$ mesopore volume, and $d_{(100)}$ value calculated by Bragg's equation $\left(\lambda=2 d_{(100)} \sin (\theta)\right), a_{\mathrm{o}}$ $=2 d_{(100)} / 3^{0.5}, t_{\mathrm{w}}=\left(a_{\mathrm{o}}-D_{\mathrm{p}}\right)$. 

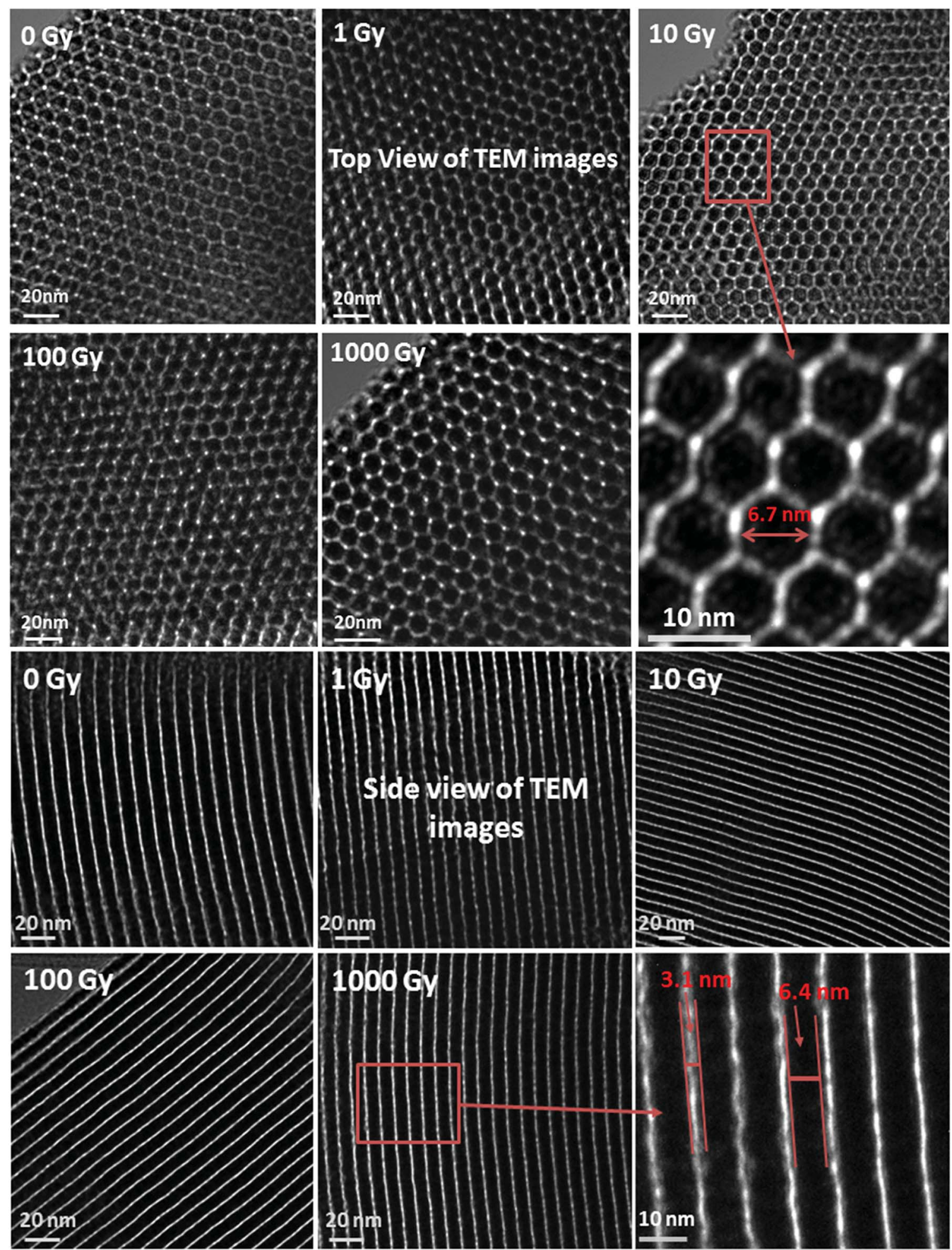

Side view of TEM images
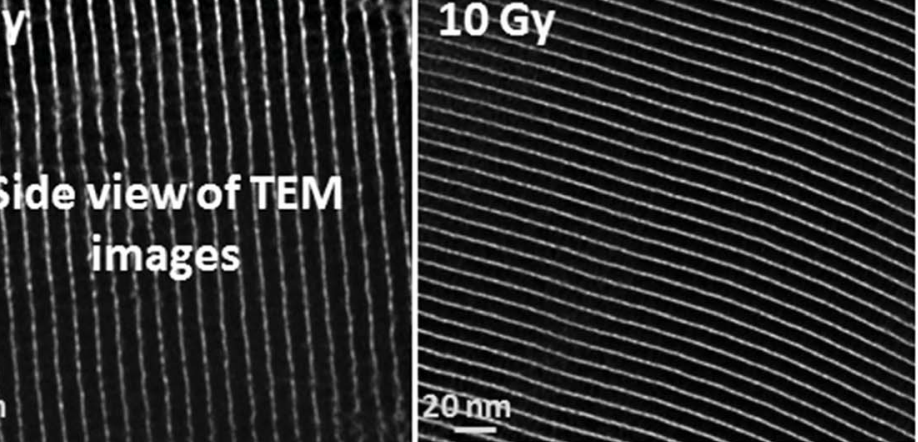

Fig. 9 SA-SBA-15 TEM images of the top view with a honeycomb-like mesoporous structure morphology and their side view of highly parallel, uniform pore and channel-like morphology as a function of gamma absorbed dose up to 1000 Gy.

protonation of the adsorbent and of ionization of the adsorbate. From Fig. 10, a negligible adsorbent capacity $\left(q_{\mathrm{e}}\right)$ was observed up to $\mathrm{pH} 4$, whereas a maximum $q_{\mathrm{e}}\left(0.34 \mathrm{mmol} \mathrm{g}^{-1}\right)$ was reached at $\mathrm{pH}$ 6. This is due to the surface protonation and the positive surface charge (Fig. 6) of the adsorbent at lower pH. After $\mathrm{pH} 4$, the positive charge of SA-SBA-15 decreases with the corresponding decrease of protonation, which leads to an increase in $q_{\mathrm{e}}$ value. $\mathrm{pH} 6$ was chosen as the optimized $\mathrm{pH}$ for this study to avoid precipitation of $\mathrm{Cu}(\mathrm{II})$ into $\mathrm{Cu}(\mathrm{OH})_{2}(\mathrm{~s})$ at $\mathrm{pH}>6$ and the excessive surface protonation at $\mathrm{pH}<6$. 


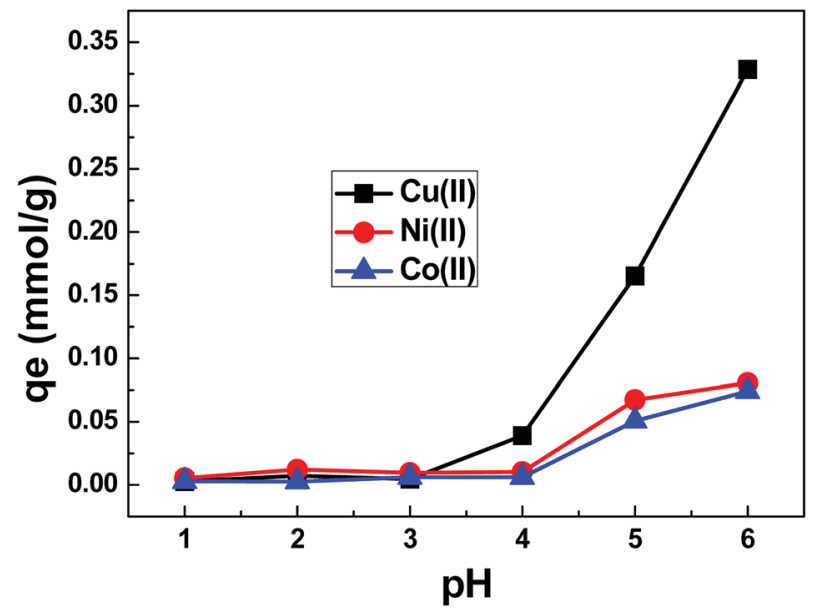

Fig. 10 SA-SBA-15 sorption capacity as a function of $\mathrm{pH}\left(C_{\mathrm{i}}=1 \mathrm{mM}\right.$, $\mathrm{S}: \mathrm{L}$ ratio $=1 \mathrm{~g} \mathrm{~L}^{-1}$, reaction time $=2 \mathrm{~h}$, ionic strength $=0.1 \mathrm{M}(\mathrm{NaCl})$ $\mathrm{HCl}$ ), shaking speed $=150 \mathrm{rpm}$ ).

$\mathrm{pH}$ was optimized in a single metal ion solution. However, the contaminated waters coming from nuclear reactor installations may contain other corrosion metals as well. The adsorbent dose needs to be optimized to capture maximum metal ions from the mixture. Therefore, the equimolar mixture $(1 \mathrm{mM}$ each) of $\mathrm{Co}(\mathrm{II}), \mathrm{Cu}(\mathrm{II})$ and $\mathrm{Ni}(\mathrm{II})$ was prepared to study the competing effects of each divalent metal ions on the sorption process. SA-SBA-15 showed a lower adsorption affinity towards the mixture of metal ions at a lower amount of adsorbent, as illustrated in Fig. 11. However, almost $100 \%$ of $\mathrm{Cu}(\mathrm{II})$ was removed from mixed metal ions at $9 \mathrm{~g} \mathrm{~L}^{-1}$ of SA-SBA-15, while 40 to $50 \%$ of $\mathrm{Co}$ (II) and $\mathrm{Ni}$ (II) was eliminated from the same mixture (Fig. 11).

The S : L ratio was optimized and batch samples were irradiated with gamma following the scheme, as shown in Fig. 2. Fig. 12 shows the percentage removal capacity $(\% R$ ) by SA-SBA15 for $\mathrm{Cu}(\mathrm{II}), \mathrm{Ni}$ (II) and $\mathrm{Co}(\mathrm{II})$ as a function of absorbed gamma dose. It is obvious from Fig. 12 that there is no negative effect of

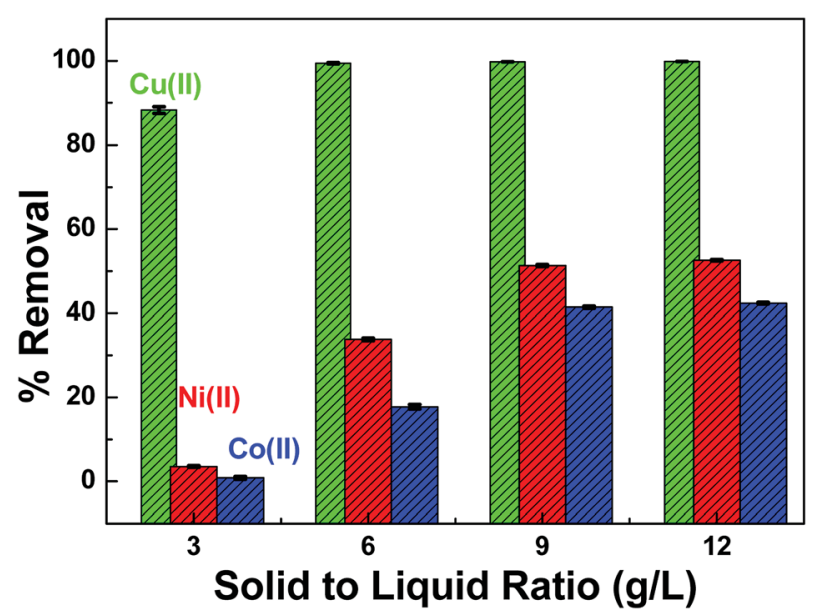

Fig. 11 Optimization of maximum removal metal ions from the equimolar mixture ( $1 \mathrm{mM}$ of each) as a function of the solid to liquid ratio (reaction time $=2 \mathrm{~h}$, ionic strength $=0.1 \mathrm{M}(\mathrm{NaCl} / \mathrm{HCl})$ ), shaking speed $=150 \mathrm{rpm}$.

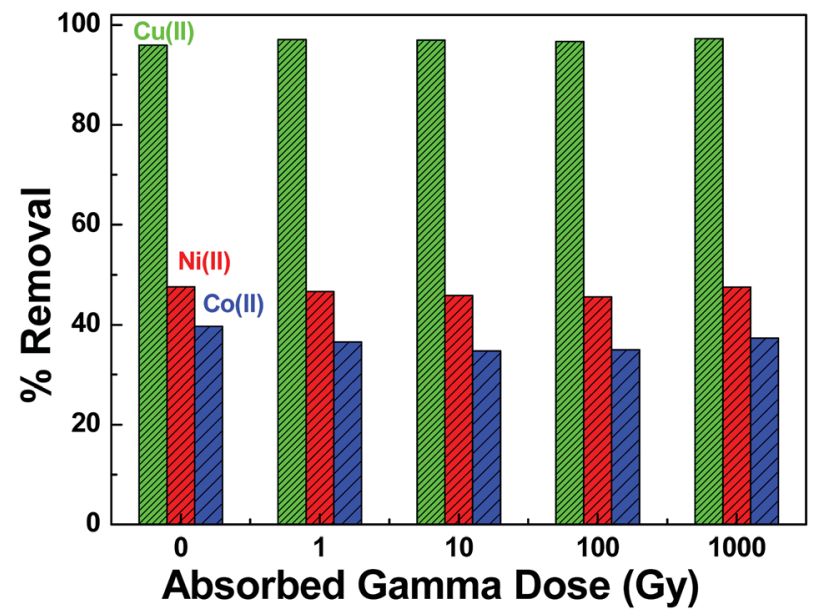

Fig. 12 Effect of gamma irradiation on the removal of $\mathrm{Cu}(॥), \mathrm{Ni}(॥)$, and Co(॥) from their mixture. (S : L ratio $=9 \mathrm{~g} \mathrm{~L}^{-1}, \mathrm{pH}=6$, ionic strength $=$ $0.1 \mathrm{M} \mathrm{HCl} / \mathrm{NaCl}$, solid-liquid contact time $=2 \mathrm{~h}$ and mechanical shaking $=150$ rpm.)

gamma irradiations on the percentage removal of metal ions by SA-SBA-15. About $97.2 \%$ of $\mathrm{Cu}(\mathrm{II}), 47.5 \%$ of $\mathrm{Ni}(\mathrm{II})$, and $37.3 \%$ of $\mathrm{Co}$ (II) were removed from solution regardless of the variation of absorbed gamma dose up to 1000 Gy. In SA-SBA-15, the bidentate Schiff's base ligand attached to the benzene ring is a potential chelating candidate to capture the metal ions from aqueous solution. ${ }^{21}$ This stability of SA-SBA-15 may be attributed to its aromaticity and conjugation which make the compounds more oxidation resistant in the presence of radiolysis products. ${ }^{\mathbf{3} 19,20}$ Moreover, the absorbed gamma dose of 1000 Gy is sufficiently enough since the dose rate in reactor core, piping areas and demineralized tank is less than $1 \mathrm{~Gy} \mathrm{~h}^{-1}$, as presented in Table 1. For the radiolytic study of organic compounds against gamma absorbed dose, 1000 Gy may be insufficient, as many organic compounds are stable at low gamma dose, except for poly-tetra-fluoro-ethylene and aliphatic polyamide (nylon), which begin to decompose at $1.5 \times 10^{2}$ and $8.7 \times 10^{2}$ Gy, respectively. ${ }^{3}$

The metal removal sequence follows the Irving-Williams order $(\mathrm{Cu}(\mathrm{II})>\mathrm{Ni}(\mathrm{II})>\mathrm{Co}(\mathrm{II}))$, which is also in good agreement to the sequence of their complexation constants of $\mathrm{Cu}(\log \beta=$ 13.0) $>\operatorname{Ni}(\log \beta=7.45)>\operatorname{Co}(\log \beta=7.13) \cdot{ }^{31,32}$ The notable stabilization of $\mathrm{Cu}$ compared to other metals ( $3 \mathrm{~d}$ series) is due to the octahedral symmetry distortion of $\mathrm{Cu}$ (II) complexes. Furthermore, the stability of the $\mathrm{Cu}(\mathrm{II})$ complexes was also confirmed by the Jahn-Teller Effect. ${ }^{31}$ Another contributing factor for the complex stability is the electronegativity of metal ions $\mathrm{Co}$ (II) $(1.88)<\mathrm{Ni}$ (II) $(1.91)<\mathrm{Cu}$ (II) (2.0). The increase in the electronegativity of metal ions enhances the covalent character of the metal-chelate bond and hence leads to the greater stability of the metal chelates. ${ }^{33}$

Adsorbent should be resistant to the gamma irradiation for decontamination of NPP coolant systems. The gamma dose rates at different locations of nuclear power plants during full power operation and shut down condition are given in Table 4 for a comparison point of view. The maximum dose rate is 100 $\mathrm{Gy} \mathrm{h}^{-1}$ at the top of the reactor core in full power operation, 
Table 4 Gamma dose rates at different locations inside the nuclear reactor power plant ${ }^{34}$

\begin{tabular}{lll}
\hline Plant locations & The dose rate at full power operation $\left(\mathrm{Gy} \mathrm{h}^{-1}\right)$ & The dose rate at shut down $\left(\mathrm{Gy} \mathrm{h}{ }^{-1}\right)$ \\
\hline Top of the reactor core & $\approx 100$ & 0.09 to 0.12 \\
Reactor coolant loops & 0.1 to 0.5 & $1 \times 10^{-4}$ to $2 \times 10^{-3}$ \\
Hot leg pipe & $\approx 2$ & $2 \times 10^{-4}$ to $2 \times 10^{-3}$ \\
Demineralized tank & $\approx 1$ & NA
\end{tabular}

while the minimum dose rate is $2 \times 10^{-4} \mathrm{~Gy} \mathrm{~h}^{-1}$ at hot leg pipelines during shut down condition. By comparing our results (Fig. 12) with that of the plant dose data in Table 4, it is clear that the synthesized adsorbent (SA-SBA-15) has very strong gamma resistance by 1 to 7 orders of magnitude. Additionally, there is no structural and functional group degradation detected before and after gamma irradiations, as observed by the FTIR, XRD, TEM, and BET results, proposing its applicability under hostile radioactive environments.

\section{Conclusions}

A highly ordered functionalized mesoporous silica (SA-SBA-15) was synthesized by a co-condensation method for the removal of activated corrosion products under gamma irradiations. Various analytical results confirmed the stability of the ligand support material, mesopore ordering and functional group under gamma irradiations up to $1000 \mathrm{~Gy}$. Results further revealed the adsorbent stability in solid and aqueous media under identical absorbed gamma dose. Our results showed a potential use of SA-SBA-15 for the decontamination and, decommissioning of nuclear power plants with excellent gamma irradiation stability both in solid as well as in liquid forms. The removal capacity for $\mathrm{Cu}$ (II) was achieved up to 99.76 $\pm 0.01 \%$. SA-SBA- 15 is more selective for $\mathrm{Cu}$ (II), which can also be exploited to separate $\mathrm{Cu}(\mathrm{II})$ from other corrosion products under the optimum conditions.

\section{Conflicts of interest}

There are no conflicts to declare.

\section{Acknowledgements}

This work was supported by the Nuclear Safety Research Program through the Korea Foundation of Nuclear Safety (KOFONS), the granted financial resource from the Nuclear Safety and Security Commission (NSSC) (No. 1305032) and by Basic Science Research Program through the National Research Foundation of Korea (NRF) funded by the Ministry of Science and ICT (No. NRF2016R1A5A1013919 and 2017M2A8A5014801), Republic of Korea.

\section{References}

1 C. C. Lin, Prog. Nucl. Energy, 2009, 51, 207-224.

2 OECD, Radiation Protection Aspects of Primary Water Chemistry and Source-term Management Report, 2014.
3 M. B. Bruce and M. V. Davis, EPRI-NP-2129, Georgia Inst. of Tech., USA, 1981.

4 J. S. Beck, J. C. Vartuli, W. J. Roth, M. E. Leonowicz, C. T. Kresge, K. D. Schmitt, C. T. W. Chu, D. H. Olson and E. W. Sheppard, J. Am. Chem. Soc., 1992, 114, 10834-10843.

5 D. Zhao, J. Feng, Q. Huo, N. Melosh, G. H. Fredrickson, B. F. Chmelka and G. D. Stucky, Science, 1998, 279, 548-552.

6 R. Sun, W. Wang, Y. Wen and X. Zhang, Nanomaterials, 2015, 5, 2019-2053.

7 K. Schumacher, P. I. Ravikovitch, A. D. Chesne, A. V. Neimark and K. K. Unger, Langmuir, 2000, 16, 4648-4654.

8 M. Schoeffel, N. Brodie-Linder, F. Audonnet and C. AlbaSimionesco, J. Mater. Chem., 2012, 22, 557-567.

9 J. Aguado, J. M. Arsuaga, A. Arencibia, M. Lindo and V. Gascón, J. Hazard. Mater., 2009, 163, 213-221.

10 J. Huang, M. Ye, Y. Qu, L. Chu, R. Chen, Q. He and D. Xu, J. Colloid Interface Sci., 2012, 385, 137-146.

11 Z. Gao, L. Wang, T. Qi, J. Chu and Y. Zhang, Colloids Surf., A, 2007, 304, 77-81.

12 J. M. Arsuaga, J. Aguado, A. Arencibia and M. S. LopezGutierrez, Adsorption, 2014, 20, 311-319.

13 E. Repo, J. K. Warchol, A. Bhatnagar, A. Mudhoo and M. Sillanpää, Water Res., 2013, 47, 4812-4832.

14 Z. Chen, Y. Wu and Y. Wei, J. Radioanal. Nucl. Chem., 2014, 299, 485-491.

15 T. Kikuchi and K. Suzuki, J. Alloys Compd., 2006, 408-412, 1287-1290.

16 K. Bernardo, S. Leppard, A. Robert, G. Commenges, F. Dahan and B. Meunier, Inorg. Chem., 1996, 35, 387-396.

17 J. R. Anacona, J. L. Rodriguez and J. Camus, Spectrochim. Acta, Part A, 2014, 129, 96-102.

18 S. A. Abdel-Latif, H. B. Hassib and Y. M. Issa, Spectrochim. Acta, Part A, 2007, 67, 950-957.

19 A. J. Swallow and A. Charlesby, Radiation Chemistry of Organic Compounds: International Series of Monographs on Radiation Effects in Materials, Elsevier Ltd., Pergamon Press, Oxford, UK, 1960, ISBN: 978-0-08-009297-3.

20 M. A. Baker, B. D. Gray, B. M. Ohlsson-Wilhelm, D. C. Carpenter and K. A. Muirhead, J. Controlled Release, 1996, 40, 89-100.

21 D. Zhao, P. Yang, N. Melosh, J. Feng, B. F. Chmelka and G. D. Stucky, Adv. Mater., 1998, 10, 1380-1381.

22 D. Zhao, J. Sun, Q. Li and G. D. Stucky, Chem. Mater., 2000, 12, 275-279.

23 S. Iqbal and J.-I. Yun, Microporous Mesoporous Mater., 2017, 248, 149-157. 
24 M. Mureseanu, A. Reiss, I. Stefanescu, E. David, V. Parvulescu, G. Renard and V. Hulea, Chemosphere, 2008, 73, 1499-1504.

25 J. Losada, I. Del Peso and L. Beyer, Inorg. Chim. Acta, 2001, 321, 107-115.

26 A. Ramirez, B. L. Lopez and L. Sierra, J. Phys. Chem. B, 2003, 107, 9275-9280.

27 M. Mureseanu, N. Cioatera, I. Trandafir, I. Georgescu, F. Fajula and A. Galarneau, Microporous Mesoporous Mater., 2011, 146, 141-150.

28 A. Rimola, D. Costa, M. Sodupe and P. Ugliengo, Chem. Rev., 2013, 113, 4216-4313.
29 P. Schneider, Appl. Catal., A, 1995, 129, 157-165.

30 C. H. Giles and D. Smith, J. Colloid Interface Sci., 1974, 47, 755-765.

31 H. Irving and R. J. P. Williams, J. Chem. Soc., 1953, 31923210.

32 R. Hernández-Molina, A. Mederos, P. Gili, S. Domínguez, F. Lloret, J. Cano, M. Julve, C. Ruiz-Pérez and X. Solans, J. Chem. Soc., Dalton Trans., 1997, 4327-4334.

33 A. A. El-Sherif, M. M. Shoukry and M. M. A. Abd-Elgawad, Spectrochim. Acta, Part A, 2012, 98, 307-321.

34 J. Sejvar, Nucl. Technol., 1977, 36, 48-55. 